doi:10.1017/\$1041610219000085

\section{A biological classification for Alzheimer's disease - Amyloid, Tau and Neurodegeneration (A/T/N): results from the Argentine-Alzheimer's Disease Neuroimaging Initiative}

Given the current controversies between the clinical and neuropathological data in Alzheimer's disease (AD), Jack et al. (2016) proposed a new biomarker based classification named as the ATN system with the goal of developing a more accurate characterization and understanding of the sequence of events that lead to cognitive impairment. Opposed to the previous concepts (Albert et al., 2011; Sperling et al., 2011), here the classification is entirely based on biomarkers (Jack et al., 2018). The seven major AD biomarkers are divided in three binary classes: "A" refers to A $\beta$ biomarkers (amyloid PET or CSF A 442 ), "T" to tau pathology biomarker (CSF p-tau or tau PET), and "N" a quantitative or topographic biomarker of neurodegeneration (CSF t-tau, FDG-PET or structural MRI) and each biomarker is rated as positive or negative (Jack et al., 2016; 2018).

The aim of this study was to describe the $\mathrm{A} / \mathrm{T} / \mathrm{N}$ research framework classification for $\mathrm{AD}$ in the Argentine ADNI cohort and to explore the prediction to develop dementia in a prospective two-year follow-up.

A cohort of 56 individuals (23 Mild Cognitive Impairment [MCI], $12 \mathrm{AD}$ and 14 controls) were studied with the ADNI-2 protocol, which has been described in detail elsewhere (Russo et al., 2014). Patients were categorized according to $\mathrm{A} / \mathrm{T} / \mathrm{N}$ and were followed longitudinally (baseline, at 12- and 24-month follow-up visits).

$\mathrm{A}+/ \mathrm{T}+/ \mathrm{N}+$ at baseline was found in $2 / 14$ controls (14\%), 2/10 early MCI (20\%), 6/13 late MCI $(46 \%)$ and $11 / 12$ mild dementia (91\%); Suspected non- AD pathophysiology (SNAP, A-/T-/N + ) was found in $1 / 14$ controls (7\%), $2 / 10$ early MCI (20\%), $2 / 13$ late $\mathrm{MCI}(15 \%)$ and $1 / 12$ mild dementia ( $8 \%)$; and A-/T-/N-: 8/14 (57\%) of normal controls, $4 / 10$ $(40 \%)$ of e-MCI, $3 / 13(23 \%)$ of $1-\mathrm{MCI}$ and $0 / 12$ $(0 \%)$ of mild dementia.

The first two-year survival analysis was based on clinical classification among normal controls $1 / 14$ (7\%), early MCI 2/10 (20\%) and late MCI 6/13 $(46 \%)$. The second two-year survival analysis was among MCI subjects according the $\mathrm{A} / \mathrm{T} / \mathrm{N}$ classification. The $A+/ T+/ N+$ subjects showed
6/8 (75\%) of conversion to DAT, A-/T- $\mathrm{N}+$ showed conversion to dementia $2 / 4(50 \%)$, and patients with $\mathrm{A}-/ \mathrm{T}-\mathrm{N}-(0 / 9)$, and $\mathrm{A}+/ \mathrm{T}-/ \mathrm{N}-(0 / 3)$ showed no conversion.

Our findings suggest that this biological classification is better for prognosis in a short time than the classical clinical one. The results should be taken with caution due to the limiting factors such as the small sample size, the short follow-up, and the source being a single specialized research center (even from ww-ADNI, the only in Latin America). However, despite all the aforementioned, these results are significant because we are the first and only ADNI center in Latin America and in a developing country with rigorous systematic with $\mathrm{AD}$ biomarkers. The present study provides a piece of evidence about the use of $\mathrm{A} / \mathrm{T} / \mathrm{N}$ classification in developing countries from Latin America. Currently, it is mandatory to use it as research framework worldwide. The $\mathrm{AD}$ biomarkers allow us to determine more detailed pathophysiology of the disease, and it is probably going to be the step to solve the appropriate therapeutics.

\section{Conflict of interest}

None.

\section{Description of authors' roles}

RFA, ES and GS designed the study, supervised the data collection and wrote the paper. IC was responsible for statistical analysis. GC, PCM, MJR, LP, PB, FT, FC, JC, FN, ES and SV collect the data and assisted with writing article.

\section{Acknowledgments}

Data collection and sharing for this project were funded by FLENI Foundation, by CONICET (Consejo Nacional de Investigaciones Cientificas y Tecnologicas - PICT 2110-2015), and by CIS (Consejo de Investigación GCBA), Argentina.

\section{References}

Albert, M. S. et al. (2011). The diagnosis of mild cognitive impairment due to Alzheimer's disease: 
recommendations from the National Institute on Aging-Alzheimer's Association workgroups on diagnostic guidelines for Alzheimer's disease. Alzheimers $\mathcal{E}$ Dementia, 7, 270-279. doi: 10.1016/j.jalz.2011.03.008.

Jack, C. R., Jr. et al. (2016). A/T/N: An unbiased descriptive classification scheme for Alzheimer disease biomarkers. Neurology, 87, 539-547. doi: 10.1212/WNL .0000000000002923 .

Jack, C. R., Jr. et al. (2018). NAI-AA research framework: toward a biological definition of Alzheimer's disease. Alzheimers \& Dementia, 14, 535-562. doi: 10.1016/j.jalz .2018.02.018.

Russo, M. J. et al. (2014). Creation of the Argentina Alzheimer disease neuroimaging initiative. Alzheimers \& Dementia, 10, S84-S87.

Sperling, R. A. et al. (2011). Toward defining the preclinical stages of Alzheimer's disease: recommendations from the National Institute on Aging-Alzheimer's Association workgroups on diagnostic guidelines for Alzheimer's disease. Alzheimers $\mathcal{E}$ Dementia, 7, 280-292. doi: 10.1016/j .jalz.2011.03.003.

Ricardo F. Allegri, ${ }^{1,2,3}$ Lucia Pertierra, ${ }^{1}$ Gabriela Cohen, ${ }^{1}$ Patricio Chrem Méndez, ${ }^{1}$ María Julieta Russo, ${ }^{1}$ Ismael Calandri, ${ }^{1}$
Pablo Bagnati, ${ }^{1}$ Fernanda Tapajóz, ${ }^{1,3}$

Florencia Clarens, ${ }^{1}$ Jorge Campos, ${ }^{1}$

Federico E. Nahas, ${ }^{1,3}$ Ezequiel Surace, ${ }^{3,4}$

Silvia VázQuez ${ }^{4,5}$ and Gustavo Sevlever ${ }^{3}$

${ }^{1}$ Department of Cognitive Neurology, Neuropsychiatry and Neuropsychology, Instituto de Investigaciones

Neurológicas FLENI, Buenos Aires, Argentina

${ }^{2}$ Department of Neurosciences, Universidad de la Costa (CUC), Barranquilla, Colombia

${ }^{3}$ CONICET (Consejo Nacional de Investigaciones Científicas y Tecnológicas), Buenos Aires, Argentina

${ }^{4}$ Department of Molecular Biology, Instituto de Investigaciones Neurológicas FLENI, Buenos Aires, Argentina

${ }^{5}$ Department of Neuroimaging, Instituto de Investigaciones Neurológicas FLENI, Buenos Aires, Argentina

Correspondence should be addressed to: Ricardo F. Allegri, Department of Cognitive Neurology. Instituto de Investigaciones Neurológicas, Montañeses 2325 (C1428AQK), Bs As, Argentina. Web: www.fleni.org.ar, Email: rallegri@fleni.org.ar 